\title{
New directions in learning and control of stochastic networks
}

\author{
Rahul Jain $^{1} \cdot$ Vijay Kamble ${ }^{2}$
}

Received: 16 December 2021 / Revised: 17 December 2021 / Accepted: 18 December 2021 /

Published online: 13 January 2022

(c) The Author(s), under exclusive licence to Springer Science+Business Media, LLC, part of Springer Nature 2022

The theory of stochastic networks and their control has a long history of applications in fields as diverse as operations research, management science, economics, and communications. Problems in this area have attracted several generations of researchers, and it remains a core defining subject in the field of applied probability. While there has always been a high activity level in this area, the theory has recently seen a particularly remarkable collaborative growth with modern machine learning and artificial intelligence techniques, fueled by many new applications.

First, there has been a surge of interest in applying machine learning techniques to the control of stochastic networks arising in modern communications applications like cloud computing and data centers. A primary goal of this pursuit has been to design agile control mechanisms that operate under minimal informational assumptions and adapt to changes in system characteristics over time. This is not exactly a new goal; indeed, researchers have been trying to understand the power and limitations of parameter-agnostic policies for network scheduling and control, such as backpressure routing and longest queue first scheduling, for many years now, for example, see [1,2] and references therein. What is new in recent pursuits is the use of modern machine learning techniques to improve upon the tradeoff frontiers achieved by existing approaches. The addition of these approaches to the toolkit has allowed for addressing challenging objectives in these systems, such as delay minimization, as well as tackling the traditional goals of utility maximization and throughput optimality in more complex settings.

Second, stochastic networks have emerged as a powerful modeling paradigm for addressing operational questions of matching demand and supply in modern platforms and marketplaces. Examples include ride-sharing platforms, labor platforms, energy markets, etc. A fundamental value proposition of these platforms is their ability to predict and mitigate supply-demand imbalances. Realizing this value requires network

\footnotetext{
Vijay Kamble

kamble@uic.edu

1 University of Southern California, Los Angeles, USA

2 University of Illinois at Chicago, Chicago, USA
} 
control mechanisms that seamlessly assimilate predictive information from data. One of the main differentiating aspects of these problems, in addition to novel network architectures and constraints, is the dependence of the success of control policies on participant input. Hence, the control policies must be robust to strategic and behavioral concerns, further complicating their design. These developments have led to a growing body of literature on the control of stochastic networks with novel architectures and at the interface of these control mechanisms with machine learning and strategic behavior. Recent special issues in QUESTA, such as "Strategic Queueing: Gametheoretic Models in Queueing Theory" (2020-2021; guest editors: A. Economou and V. Kulkarni) and "Stochastic Matching Models, Matching Queues and Applications" (2021; guest editors: J. Mairesse and P. Moyal), reflect these exciting developments.

We invite researchers to consider QUESTA as a forum for the quick dissemination of new ideas in this space. We are happy to present an excellent paper in this direction by W. Weng and R. Srikant that was submitted to the Special Issue on "New directions in learning and control of stochastic networks," and we expect more such papers to come to the journal.

\section{On behalf of Guest Editors:}

Rahul Jain (University of Southern California)

Vijay Kamble (University of Illinois at Chicago)

Sanjay Shakkottai (University of Texas at Austin)

Jiaming Xu (Duke University)

\section{References}

1. Srikant, R.: The Mathematics of Internet Congestion Control. Springer (2004)

2. Jiang, L., Walrand, J.: Scheduling and congestion control for wireless and processing networks. Synth. Lect. Commun. Netw. 3(1), 1-156 (2010)

Publisher's Note Springer Nature remains neutral with regard to jurisdictional claims in published maps and institutional affiliations. 